\title{
Reliability of Histopathology for the Early Recognition of Fibrosis in Traction Alopecia: Correlation with Clinical Severity
}

\author{
Reginald Mzudumile Ngwanya ${ }^{a}$ Henry Ademola Adeola ${ }^{a}$ \\ Renée A. Beach ${ }^{b}$ Nomphelo Gantsho ${ }^{a}$ Christopher L. Walker ${ }^{c}$ \\ Komala Pillayc Robert Prokopetz ${ }^{\text {b }}$ Freedom Gumedze $^{d}$ \\ Nonhlanhla P. Khumaloa \\ ${ }^{a}$ Division of Dermatology, Faculty of Health Sciences, University of Cape Town, \\ Cape Town, South Africa; ${ }^{b}$ Division of Dermatology and Pathology, University of

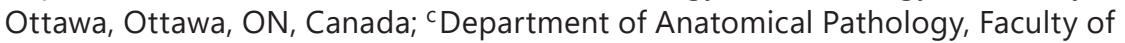 \\ Health Sciences, University of Cape Town, Cape Town, South Africa; ${ }^{d}$ Department of \\ Statistical Sciences, University of Cape Town, Cape Town, South Africa
}

\section{Keywords}

African hair · Afro-textured hair · Afro-American hair · Traction alopecia · Minoxidil ·

Dermatopathology

\begin{abstract}
Traction alopecia (TA) is hair loss caused by prolonged pulling or repetitive tension on scalp hair; it belongs to the biphasic group of primary alopecia. It is non-scarring, typically with preservation of follicular stem cells and the potential for regrowth of early lesions especially if traction hairstyles are stopped. However, the alopecia may become permanent (scarring) and fail to respond to treatment if the traction is excessive and prolonged. Hence, the ability to detect fibrosis early in these lesions could predict patients who respond to treatment. Histopathological diagnosis based on scalp biopsies has been used as a gold standard to delineate various forms of non-scarring alopecia and to differentiate them from scarring ones. However, due to potential discrepant reporting as a result of the type of biopsy, method of sectioning, and site of biopsy, histopathology often tends to be unreliable for the early recognition of fibrosis in TA. In this study, 45 patients were assessed using the marginal TA severity scoring system, and their biopsies (both longitudinal and transverse sections) were systematically assessed by three dermatopathologists, the aim being to correlate
\end{abstract}


Ngwanya et al.: How Scarring Is Traction Alopecia

histopathological findings with clinical staging. Intraclass correlation coefficients were used to determine the level of agreement between the assessors. We found poor agreement of the identification and grading of perifollicular and interfollicular fibrosis $(0.55$ [0.23-0.75] and 0.01 [2.20-0.41], respectively), and no correlation could be drawn with the clinical severity score. Better methods of diagnosis are needed for grading and for recognition of early fibrosis in TA.

(C) 2019 The Author(s)

Published by S. Karger AG, Basel

\section{Introduction}

Traction alopecia (TA) is a form of acquired hair loss that results from prolonged pulling or repetitive tension on scalp hair [1]. It is the commonest form of hair loss affecting about $30 \%$ of African women [2,3]. TA was found to be present in $17.1 \%$ of school girls and in $31.7 \%$ of females older than 18 years of age [2]. It is caused by chronic traction from hairstyles (braids, pulled back hair, weaves, and the use of elastic bands for the hair); the risk is higher with traction on chemically relaxed hair [2]. The location of TA wholly depends on an individual's hair care practice and mostly affects the front hairline and sides of the scalp. The clinical spectrum of TA ranges from acute to chronic, and it is considered a non-inflammatory, non-scarring, biphasic form of alopecia (i.e., initially reversible but can become scarring in chronic disease) [4]. The fringe sign, which is the retention of hair along the marginal hairline, is often observed in TA [5].

Although TA has been well characterized clinically, its histopathological features are poorly characterized. For example, it can be challenging to delineate TA from other types of non-scarring alopecia, such as alopecia areata in the absence of the classical peribulbar inflammation seen in this type of alopecia [6]. Classically, TA is characterized by a reduction in the number of terminal hair follicles with a normal amount of vellus hair [6].

Currently, semi-quantitative parameters, such as the terminal-to-vellus hair ratio, percentage of telogen hair, and follicular density determined using transverse scalp biopsy sections [7], have been used in combination with histomorphological assessment to differentiate TA from other forms of alopecia [7, 8]. Histologically, early features include perifollicular and intrafollicular haemorrhage, as well as the presence of pigment casts $[9,10]$. Also, hair follicle numbers are normal; however, there is an increase in catagen/telogen follicles. The late stage shows decreased terminal hair follicles with preservation of vellus hair follicles and sebaceous glands $[11,12]$. Follicular units at the level of the isthmus are replaced by fibrosis, and, hence, permanent alopecia ensues. Although features of late-stage scarring are well characterized clinicopathologically in TA, there are no known clinical features that correlate with the histopathological features of scarring that develop in chronic alopecia areata, further making them difficult to delineate. The marginal TA severity (M-TAS) scale is a clinical grading tool which has been developed to provide an objective method to grade the severity of TA [3]. Although not intended as a diagnostic tool, M-TAS was developed as a severity scoring system for marginal TA and to test its reliability.

Against this backdrop, this study aims to determine the reliability of histopathological grading for the early recognition of fibrosis in TA and to determine whether it is possible to correlate clinical presentation with the severity of histopathological scarring - given that TA is biphasic histopathologically. This could guide the clinician in deciding which patients are offered early treatment as later stage is permanent [13]. We also wanted to know if there would be good interobserver correlation in assessing histopathological findings. In this study, we have used M-TAS as a clinical grading tool that will be correlated with the histopathological findings of fibrosis in TA. 
Ngwanya et al.: How Scarring Is Traction Alopecia

\section{Materials and Methods}

\section{Study Design}

This is a prospective, observational study of 45 women who were clinically diagnosed as having TA (details are listed in the online suppl. Table; for all online suppl. material, see www.karger.com/doi/10.1159/000500509).

\section{Study Participant Selection}

Participants enrolled for this study were healthy adults who were older than 18 years and were recruited from University of Cape Town students, the staff of the Groote Schuur Hospital, and visitors accompanying patients to the Groote Schuur Hospital in Cape Town, South Africa. A total of 45 patients were graded for severity of TA using the M-TAS severity grading system (Fig. 1). Permission was granted for taking longitudinal and cross-section biopsies of the scalp, which were independently and systematically assessed by 3 pathologists.

\section{Inclusion Criteria}

All participants have Afro-textured hair (which can be defined as hair that originates from a curved hair follicle and is tightly curly or has spiral curls). Only TA cases diagnosed by a dermatologist were included. The participants did not have any hair colour or dye applied within the previous 6 weeks of the biopsy. Also, the participants were not wearing hair extensions/braid extensions, hair weaves, or other forms of artificial hair cosmesis. The last reported application of relaxer to the participants' hair was at least 2 weeks prior to the study, they were not using any prescription medications that may affect hair growth or loss, and they are not under any medical treatment. All selected participants were able to complete a hair history questionnaire and were able to provide informed consent to participate in the study.

\section{Standardized Photography}

Photographs of the participants' hairline were taken without any identifying physical or facial features at each visit. The identifier to match patient intake form, photo, and biopsy samples consisted of a number code assigned to each participant to de-identify them.

\section{Hair Loss Severity}

For the development of M-TAS, margins of the scalp were divided into anterior and posterior. Both anterior and posterior regions were further subdivided into 3 areas using the temporalis muscle and the mastoid process as landmarks. A total of 6 areas were identified, which were each assessed by the examiner [3]. Large interclass correlation coefficients were previously observed, and interobserver agreement was reported to be substantial to excellent for both anterior and posterior areas [3]. Investigator assessment was performed using validated M-TAS scores, and standard photography was used for comparisons made by 3 blinded assessors.

\section{Procedure on Visits}

Visit 1 (Day 1)

An area affected by TA was chosen in each patient. Standard 4-mm "punch" biopsies were performed on the selected areas under aseptic conditions and local anaesthetic (lignocaine). The sites were sutured with two 5.0 nylon sutures and covered with a bandage.

Visit 2 (Day 8)

Eight days later, the sutures were removed by a study doctor, and the site was inspected for appropriate wound healing. 
Hair Clinic

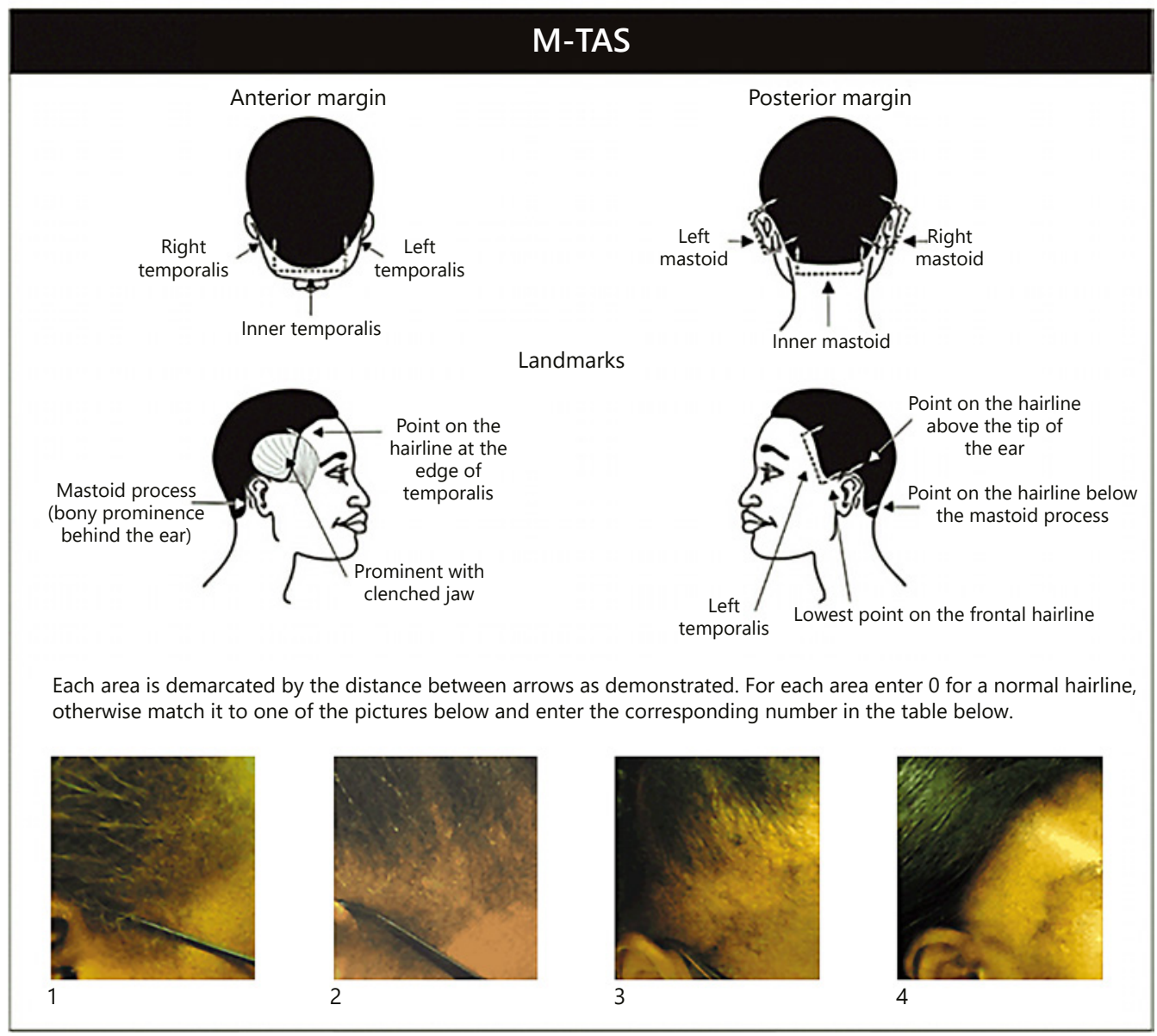

Patient name:

Folder No.

\begin{tabular}{|l|l|l|l|l|l|l|l|l|l|l|}
\hline Date & & & & & & & & & & \\
\hline $\begin{array}{l}\text { Right } \\
\text { temporalis }\end{array}$ & & & & & & & & & & \\
\hline $\begin{array}{l}\text { Inner } \\
\text { temporalis }\end{array}$ & & & & & & & & & & \\
\hline $\begin{array}{l}\text { Left } \\
\text { temporalis }\end{array}$ & & & & & & & & & & \\
\hline $\begin{array}{l}\text { Total anterior } \\
\text { score }\end{array}$ & & & & & & & & & & \\
\hline $\begin{array}{l}\text { Right } \\
\text { mastoid }\end{array}$ & & & & & & & & & & \\
\hline $\begin{array}{l}\text { Inner } \\
\text { mastoid }\end{array}$ & & & & & & & & & & \\
\hline $\begin{array}{l}\text { Left } \\
\text { mastoid }\end{array}$ & & & & & & & & & & \\
\hline $\begin{array}{l}\text { Total posterior } \\
\text { score }\end{array}$ & & & & & & & & & & \\
\hline
\end{tabular}

Fig. 1. The marginal traction alopecia severity score (M-TAS) showing regions that are assessed to provide an objective assessment of severity grading for traction alopecia (reproduced with permission from Khumalo et al. [3]). 


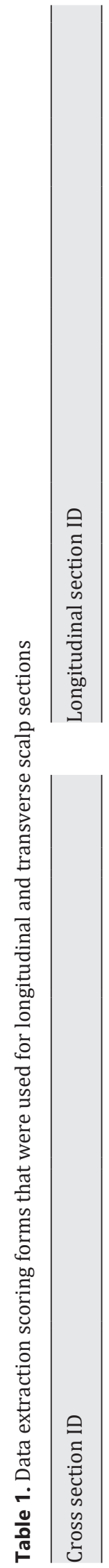

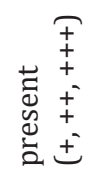

$\vec{\Xi}$
$\stackrel{\tilde{\omega}}{\sigma}$

$\stackrel{5}{\underbrace{\infty}}$

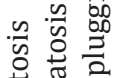

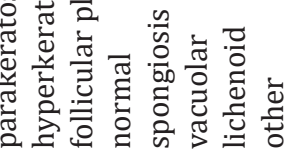

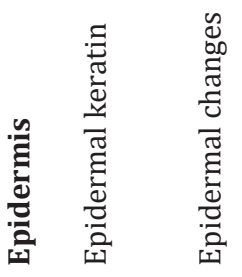

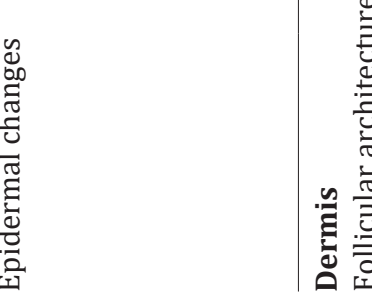

$\stackrel{\infty}{\stackrel{D}{D}}$
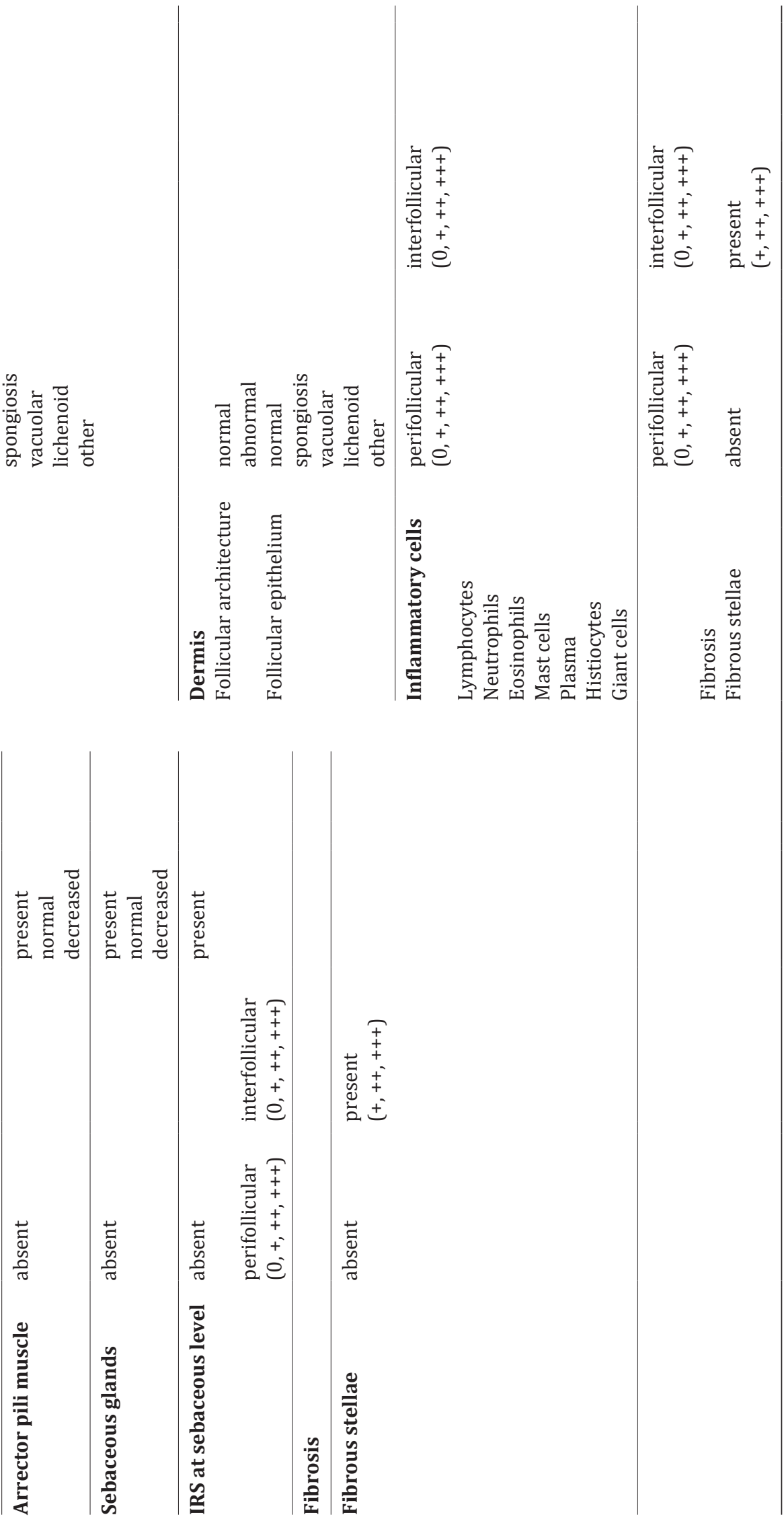

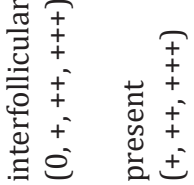

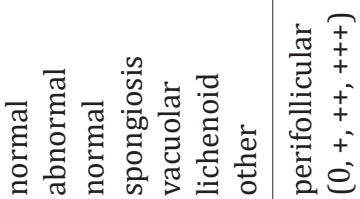

KARGER 


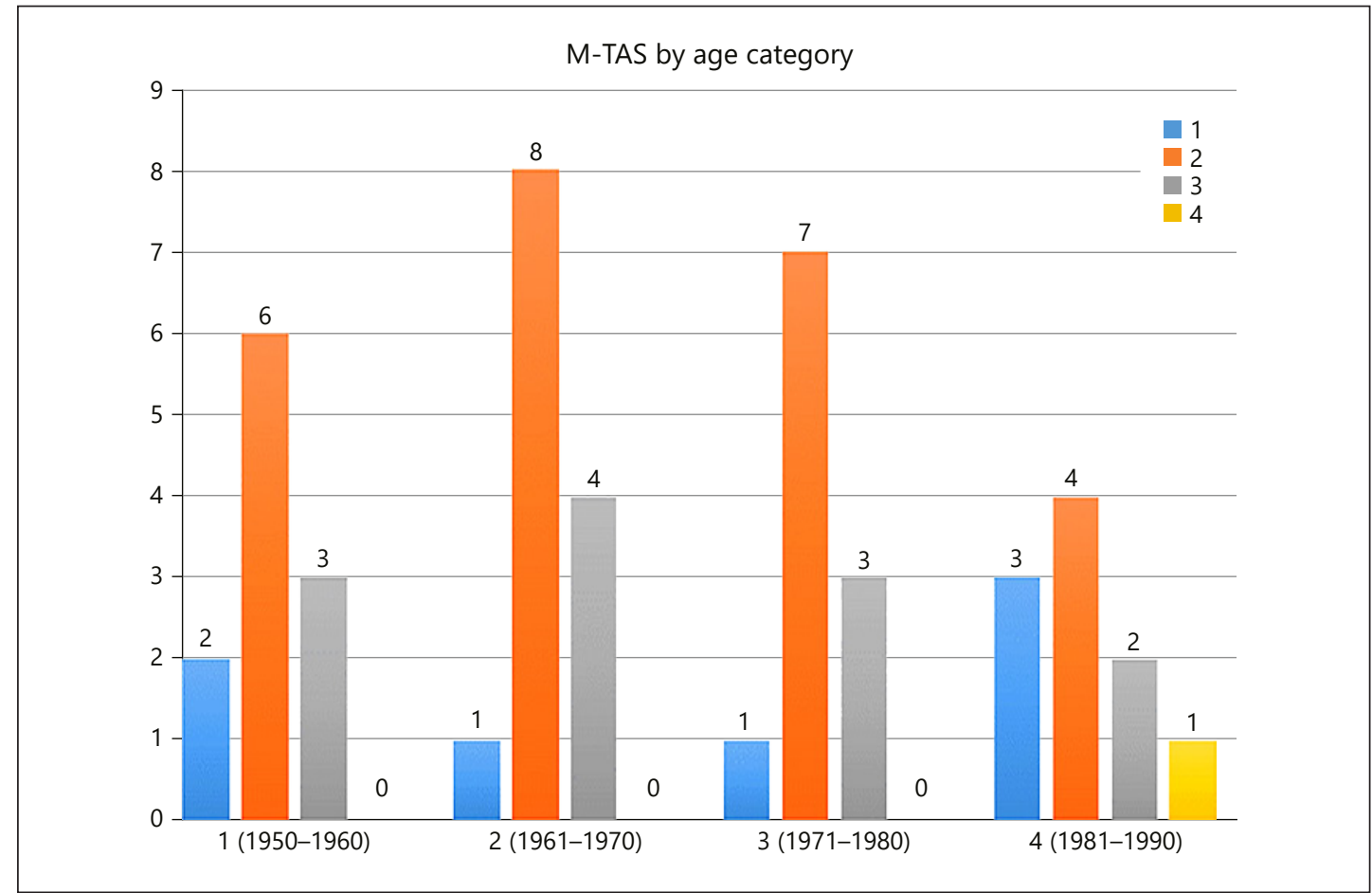

Fig. 2. Bar graph showing clinical severity scoring of traction alopecia (TA) for 45 participants using the M-TAS score. The stage 2 score was very high in all 4 decades. Stage 4 TA was not detected in participants born between 1950 and 1980; however, a participant born between 1980 and 1990 had stage 4 .

\section{Histopathology}

All scalp biopsy specimens taken on day 1 were processed into formalin-fixed, paraffinembedded sections, stained with haematoxylin and eosin (H\&E), and assessed by an experienced dermatopathologist who had no clinical examination or experience with the patients. The dermatopathologist assessed signs of scarring alopecia or fibrosis at the site of the pilo-sebaceous follicles using standardized guidelines for both the longitudinal and transverse sections of the scalp biopsy (Table 1). Even with the use of special stains such as elastic van Gieson (EVG) and Masson trichrome (MT), which are used to identify elastic and collagen fibres, respectively, we could not clearly delineate between early and late TA (Fig. 2).

\section{Analysis of Data}

Data generated were analysed using intraclass correlation coefficient (ICC) to determine the level of agreement between the assessors. The ICC scores were interpreted as shown in Table 2.

\section{Results}

\section{Age Distribution}

For all 45 participants, the birth year ranged from 1950 to 1989, and the mean year of birth was 1971. Age distribution between the 4 decades $(1950,1960,1970$, and 1980) was even (Table 3); the decade with the highest frequency was 1961-1970 (28.89\%). 
Table 2. Intraclass correlation coefficient (ICC) analysis for traction alopecia
Ngwanya et al.: How Scarring Is Traction Alopecia

\begin{aligned} & \hline Score Interpretation \\ & \hline $0-0.2$ poor agreement \\ & $0.3-0.4$ fair agreement \\ & $0.5-0.6$ moderate agreement \\ & $0.7-0.8$ strong agreement \\ &$>0.8$ almost perfect agreement \\ & \hline\end{aligned}

High interrater reliability with an ICC of 1 denotes perfect agreement and 0 mean random agreement.
Table 3. Age distribution among the 45 participants with traction alopecia

\begin{tabular}{lll}
$\begin{array}{l}\text { Age range: } 23-63 \text { years } \\
\text { Mean age: } 42 \text { years }\end{array}$ & \\
\hline Age & $n$ & $\%$ \\
\hline 1 (53-63 years) & 11 & 24.44 \\
2 (43-52 years) & 13 & 28.89 \\
$3(33-42$ years) & 11 & 24.44 \\
$4(23-32$ years) & 10 & 22.22 \\
\hline Total & 45 & 99.99 \\
\hline
\end{tabular}

\section{Clinical TA Severity Using the M-TAS Score}

Among the 45 patients evaluated in this study, it was found that stage 2 clinical severity was the commonest across the 4 decades. Also, stage 4 severity was not found in participants except for 1 case of stage 4 TA in the 1980 decade (Fig. 2).

\section{Special Histopathological Staining}

Even with the use of special stains such as EVG and MT, which are used to identify elastic and collagen fibres, respectively, we could not clearly delineate between early and late TA (Fig. 3).

\section{Histopathological Scoring for TA}

Follicular Architecture

Using the transverse cross sections, extruded hair shafts in the perifollicular and interfollicular regions had a good interclass correlation of 0.91 (0.84-0.95) and $0.83(0.75-0.91)$, respectively (Fig. 4a). Interclass correlations found in follicular architecture using longitudinal sections were poor $(0.12$ [0.0-0.52]) and unreliable (Fig. 4b).

Sebaceous Glands

A fair interclass correlation $(0.70$ [0.48-0.84]) was found between clinical staging of TA and the presence of sebaceous glands (Fig. 5).

Follicular Fibrosis

A poor interclass correlation was noted for interfollicular fibrosis $(0.01[2.20-0.41])$ and the presence of fibrous stellae $(0.01$ [ -0.98 to 0.34$])$. A relatively poor interclass correlation (0.55 [0.23-0.75]) was found for the presence of perifollicular fibrosis as well (Fig. 5). 

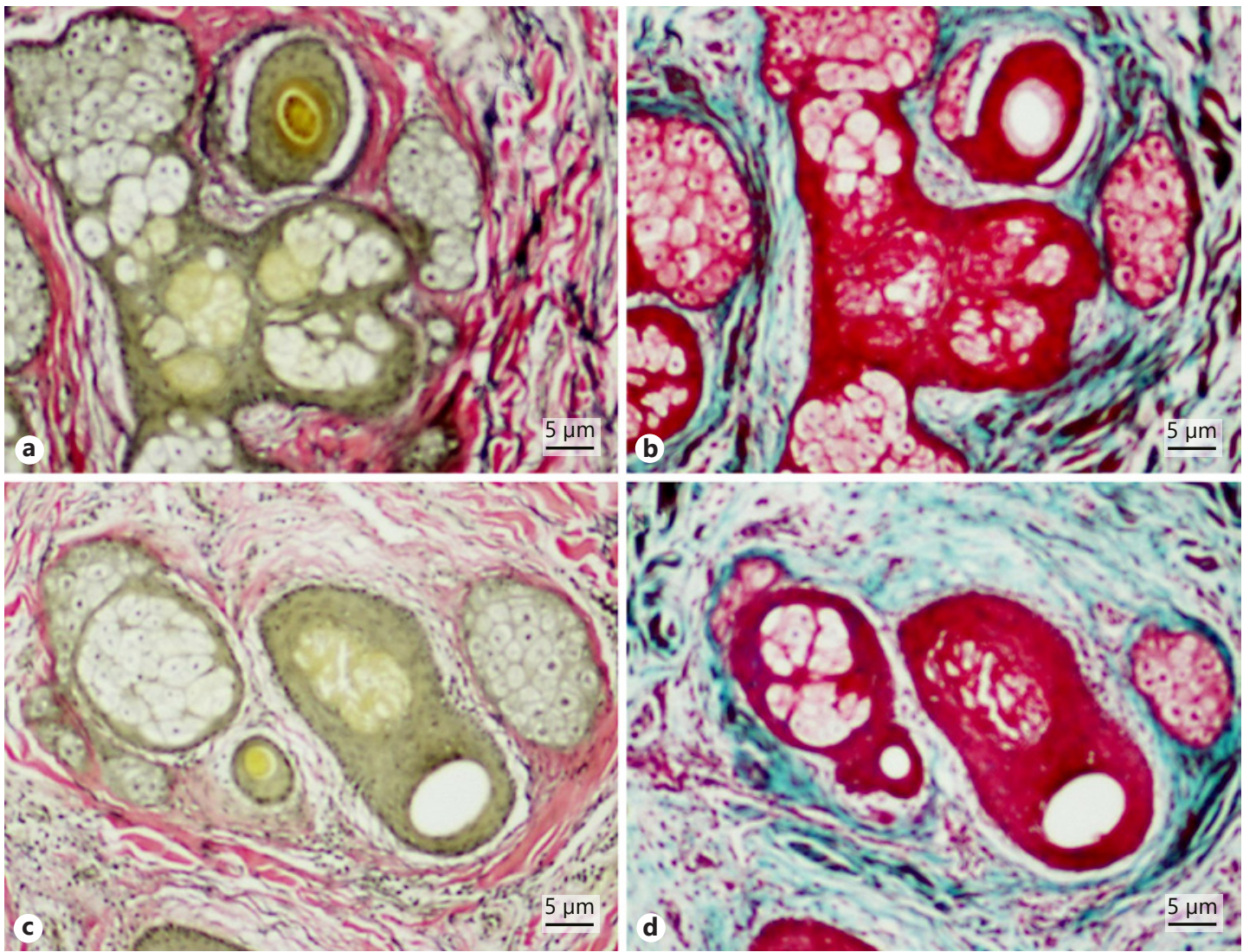

Fig. 3. Elastic van Gieson (EVG) and Masson trichrome (MT) special stains $(\times 10)$ for early $(\mathbf{a}, \mathbf{b})$ and late traction alopecia (TA) (c, d). EVG shows elastic fibres on both early and late TA. MT shows thick collagen fibres. TA severity could neither be delineated using EVG $(\mathbf{a}, \mathbf{c})$ nor MT $(\mathbf{b}, \mathbf{d})$. There is loss of elastic fibres and perifollicular fibrosis in late TA (c, d).

\section{Discussion and Conclusion}

TA is a disease that affects a significant proportion of African women [3]. Therefore, this study is focused on testing the reliability of histopathological findings correlated with the clinical grading system for TA. We found here that there were more patients with an M-TAS score of 2 and only 1 with a severe or high score irrespective of age. This may indicate that the disease lingers considerable at the early phase before progressing to the late irreversible stages. This also alludes to the benefit of early diagnosis and reversal of TA-precipitating hair grooming and styling habits. It has been reported that the prognosis of TA is variable depending on how early the traction on the hair is stopped [14]. If the hair technique that exerts traction is stopped early, there can be reversal of the clinical phenotype; however, if traction continues for a prolonged period, chronic perifollicular inflammation may lead to permanent scarring alopecia [14]. We also found good agreement between the identification of extruded hair shafts and the presence of sebaceous glands. Early-phase TA has been characterized by the presence of trichomalacia and increased presence of catagen and telogen hairs, which is then followed by "follicular abandonment," loss of terminal hair, retention of vellus hair, and sebaceous glands with decreased sizes $[14,15]$. Hence, as seen in this study, a decrease in the size of sebaceous glands may be a surrogate indicator for the progression of TA; in contrast with other conditions like telogen effluvium, in which the terminal/vellus hair ratio remains the 
Fig. 4. Photomicrographs of the follicular architecture of traction alopecia. a Presence of peri- and interfollicular scarring surrounding extruded, naked fragment of hair shafts. b Loss of normal follicular architecture in a longitudinal section of scalp biopsy.

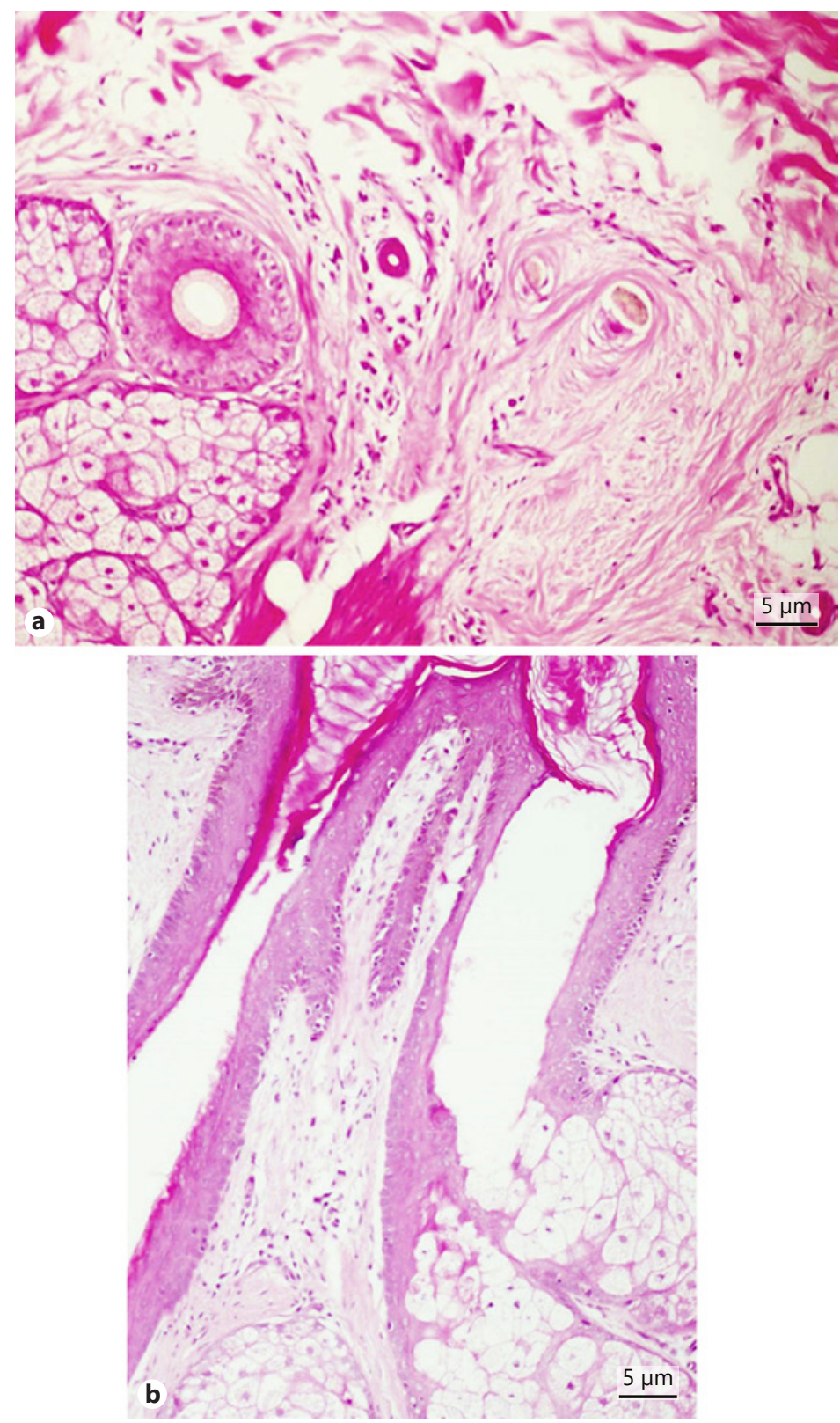

same, and there is no alteration in the sebaceous gland volume [7]. However, solely relying on a decrease in sebaceous glands is unreliable as loss of sebaceous glands can be frequently found in some primary cicatricial alopecias, such as central centrifugal cicatricial alopecia [15]. Not least, the volume of sebaceous glands varies, depending on the level of sectioning, as the volume of sebaceous glands reduces in size from the isthmus to the lower infundibulum.

Even though there was good agreement between pathologists on parameters such as the extrusion of hair shafts and the presence of sebaceous glands, we observed that there was poor agreement between observers on the identification and grading of the severity of fibrosis. Even though not restricted to TA, a fibrotic phenotype in the advanced stages of TA [14] could be a potential indicator of disease progression, albeit perifollicular fibrosis is able to differentiate TA from other traction-induced conditions, such as trichotillomania, which is reported to present with empty anagen hair follicles, increased catagen hairs without fibrosis, 
Fig. 5. Photomicrographs showing fibrosis in transverse sections of traction alopecia (TA). a Follicular scar in end-stage permanent chronic TA with fibrosis at the isthmic level. b Thickened perifollicular fibrosis, loss of sebaceous glands, and inflammation.

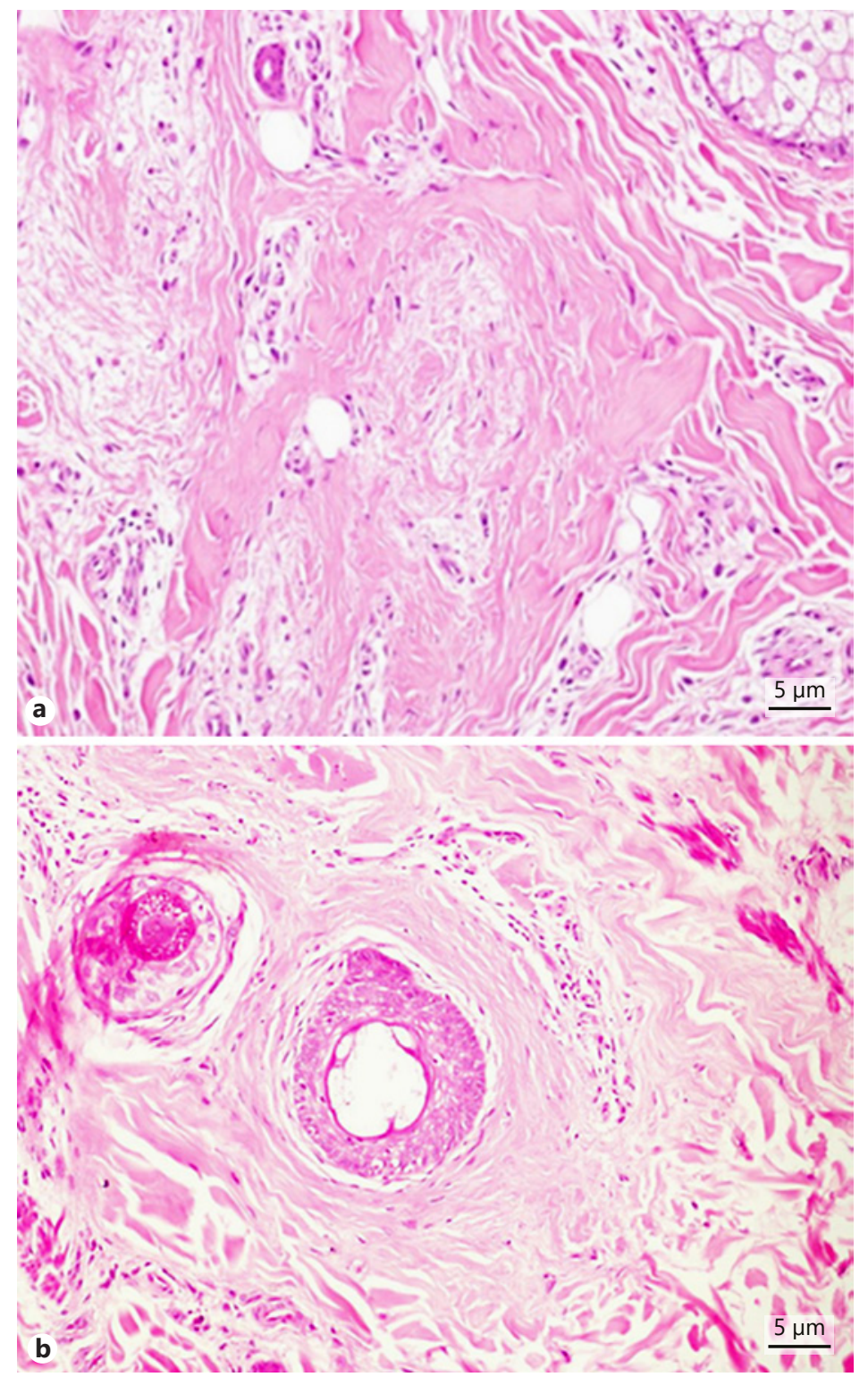

or inflammation [10]. In addition, assessment of the extent of fibrosis by physical eye may be biased, and novel tools are needed to quantitatively assess the severity of fibrosis in TA. As it is apparent that we could not differentiate fibrosis in early or late TA, it is imperative that molecular tools that can identify and quantitate fibrosis are developed. For example, for liver fibrosis, conventional histological category systems applied to describe fibrotic changes in the liver are deemed incapable of reflecting fibrosis dynamics with reproducibility and sufficient precision [16]. Other novel tools have been developed to reliably quantify the degree of fibrosis, and this has improved the management of fibrotic liver diseases tremendously [1618]. Furthermore, our study found that there was poor agreement and correlation between the diagnosed degree of fibrosis in the TA samples, and no correlation could be drawn with the clinical severity score (using the M-TAS grading). This indicates that histopathological diagnosis does not necessarily correlate with the clinical phenotype, and that non-invasive molecular approaches may be more appropriate for the diagnosis of TA severity. 
Being able to detect fibrosis at an early stage is of great importance in deciding which patient is likely to benefit from treatment (such as minoxidil). In late stages, it is well known that chronic TA is capable of setting severe folliculitis in motion and subsequently results in scarring and permanent hair loss, albeit early TA is amenable to the use of minoxidil [19]. Our study has shown that there is a pressing need to improve the degree of agreement in identification and grading of fibrosis among dermatopathologists. Exciting new tools for early recognition and grading of fibrosis such as Fourier transform infrared (FTIR) spectroscopy are needed. FTIR spectroscopy is based on using selective infrared-red adsorption of different molecules for biochemical identification. FTIR has been used to assess fibrillary collagen deposition in myocardial infarction [20]; renal graft fibrosis [21], and various other pathologies [22].

In conclusion, we have seen from this study that histopathological assessment of scalp biopsy may not be the best tool for the assessment of early fibrosis in TA, although it proved dependable when assessing cross-sectional follicular architecture and retention of sebaceous glands. A poor correlation was found with perifollicular fibrosis, interfollicular fibrosis, and the presence of fibrous stellae. Novel molecular tools are needed for early recognition of fibrosis, particularly in Africa, where various hair grooming and dressing practices have led to a high prevalence of TA.

\section{Acknowledgment}

H.A.A. thanks the South African Medical Research Council (SAMRC) for a mid-career scientist and self-initiated research grant and the South African National Research Foundation (NRF) for incentive and research development grants for rated researchers. N.P.K. thanks the SAMRC and the South African NRF Research Chair Initiative.

\section{Statement of Ethics}

The authors declare that all the patients have given their written informed consent, and the study protocol has been approved by the University of Cape Town Research Ethics Committee.

\section{Disclosure Statement}

The authors have no conflicts of interest to declare.

\section{Author Contributions}

N.P.K. conceptualized the study. R.M.N., H.A.A., and N.P.K. drafted and revised the manuscript. R.M.N. and H.A.A. were responsible for the micro-images. R.A.B. and N.G. recruited, examined, and biopsied the patients, and drafted the manuscript. C.L.W., K.P., R.M.N., and R.P. reviewed the slides, C.L.W., K.P., and R.M.N., provided histopathologic diagnosis and approved the manuscript. F.G. provided statistical analysis and revised the manuscript. 


\section{References}

1 Xu L, Liu KX, Senna MM. A Practical Approach to the Diagnosis and Management of Hair Loss in Children and Adolescents. Front Med (Lausanne). 2017 Jul;4:112.

2 Khumalo NP, Jessop S, Gumedze F, Ehrlich R. Determinants of marginal traction alopecia in African girls and women. J Am Acad Dermatol. 2008 Sep;59(3):432-8.

3 Khumalo NP, Ngwanya RM, Jessop S, Gumedze F, Ehrlich R. Marginal traction alopecia severity score: development and test of reliability. J Cosmet Dermatol. 2007 Dec;6(4):262-9.

4 Akingbola CO, Vyas J. Traction alopecia: A neglected entity in 2017. Indian J Dermatol Venereol Leprol. 2017 Nov-Dec;83(6):644-9.

5 Samrao A, Price VH, Zedek D, Mirmirani P. The "Fringe Sign" - A useful clinical finding in traction alopecia of the marginal hair line. Dermatol Online J. 2011 Nov;17(11):1.

6 Bernárdez C, Molina-Ruiz AM, Requena L. Histologic features of alopecias-part I: nonscarring alopecias. Actas Dermosifiliogr. 2015 Apr;106(3):158-67.

7 Werner B, Mulinari-Brenner F. Clinical and histological challenge in the differential diagnosis of diffuse alopecia: female androgenetic alopecia, telogen effluvium and alopecia areata - part II. An Bras Dermatol. 2012 Nov-Dec;87(6):884-90.

8 Werner B, Mulinari-Brenner F. Clinical and histological challenge in the differential diagnosis of diffuse alopecia: female androgenetic alopecia, telogen effluvium and alopecia areata - part I. An Bras Dermatol. 2012 Sep-Oct;87(5):742-7.

9 Barbosa AB, Donati A, Valente NS, Romiti R. Patchy Traction Alopecia Mimicking Areata. Int J Trichology. 2015 Oct-Dec;7(4):184-6.

10 Emer J, Luber A, Gropper J, Sidhu H, Phelps R. A patch of hair loss on the scalp. J Clin Aesthet Dermatol. 2013 Jul;6(7):45-9.

11 Herskovitz I, Miteva M. Central centrifugal cicatricial alopecia: challenges and solutions. Clin Cosmet Investig Dermatol. 2016 Aug; 9:175-81.

12 Fung MA, Sharon VR, Ratnarathorn M, Konia TH, Barr KL, Mirmirani P. Elastin staining patterns in primary cicatricial alopecia. J Am Acad Dermatol. 2013 Nov;69(5):776-82.

13 Khumalo NP, Ngwanya RM. Traction alopecia: 2\% topical minoxidil shows promise. Report of two cases. J Eur Acad Dermatol Venereol. 2007 Mar;21(3):433-4.

14 Tanus A, Oliveira CC, Villarreal DJ, Sanchez FA, Dias MF. Black women's hair: the main scalp dermatoses and aesthetic practices in women of African ethnicity. An Bras Dermatol. 2015 Jul-Aug; 90(4):450-65.

15 Qi J, Garza LA. An overview of alopecias. Cold Spring Harb Perspect Med. 2014 Mar;4(3):4.

16 Wang Y, Hou JL. Current strategies for quantitating fibrosis in liver biopsy. Chin Med J (Engl). 2015 Jan;128(2): 252-8.

17 Almpanis Z, Demonakou M, Tiniakos D. Evaluation of liver fibrosis: “Something old, something new...” Ann Gastroenterol. 2016 Oct-Dec;29(4):445-53.

18 Papastergiou V, Tsochatzis E, Burroughs AK. Non-invasive assessment of liver fibrosis. Ann Gastroenterol. 2012;25(3):218-31.

19 Mounsey AL, Reed SW. Diagnosing and treating hair loss. Am Fam Physician. 2009 Aug;80(4):356-62.

20 Cheheltani R, Rosano JM, Wang B, Sabri AK, Pleshko N, Kiani MF. Fourier transform infrared spectroscopic imaging of cardiac tissue to detect collagen deposition after myocardial infarction. J Biomed Opt. 2012 May; 17(5):056014.

21 Vuiblet V, Fere M, Gobinet C, Birembaut P, Piot O, Rieu P. Renal Graft Fibrosis and Inflammation Quantification by an Automated Fourier-Transform Infrared Imaging Technique. J Am Soc Nephrol. 2016 Aug;27(8): 2382-91.

22 Sreedhar H, Varma VK, Nguyen PL, Davidson B, Akkina S, Guzman G, et al. High-definition Fourier Transform Infrared (FT-IR) spectroscopic imaging of human tissue sections towards improving pathology. J Vis Exp. 2015 Jan;(95):52332. 